\title{
Modeling and Integrating Losses of Magnetic Components Into Time-Domain Electric Circuit Simulations
}

N. Djekanovic, M. Luo, and D. Dujic

This material is posted here with permission of the IEEE. Such permission of the IEEE does not in any way imply IEEE endorsement of any of EPFL's products or services. Internal or personal use of this material is permitted. However, permission to reprint / republish this material for advertising or promotional purposes or for creating new collective works for resale or redistribution must be obtained from the IEEE by writing to pubs-permissions@ieee. org. By choosing to view this document, you agree to all provisions of the copyright laws protecting it. 


\section{Modeling and Integrating Losses of Magnetic Components Into Time-Domain Electric Circuit Simulations}

\author{
Nikolina Djekanovic \\ Power Electronics Laboratory \\ EPFL \\ Lausanne, Switzerland \\ nikolina.djekanovic@epfl.ch
}

\author{
Min Luo \\ Plexim $\mathrm{GmbH}$ \\ Zurich, Switzerland \\ luo@plexim.com
}

\author{
Drazen Dujic \\ Power Electronics Laboratory \\ EPFL \\ Lausanne, Switzerland \\ drazen.dujic@epfl.ch
}

\begin{abstract}
To shorten converter design time and maximize the cost efficiency, advanced design tools are needed. These tools need to be capable of performing simulations in multiple relevant converter domains, such as electric, magnetic, dielectric, and thermal, simultaneously. This paper presents a verified way to calculate semiconductor, magnetic core, and winding losses in a common simulation environment. For validation purposes, a circuit combining a full-bridge LLC resonant converter galvanically isolated through a transformer is simulated. The transformer model is based on electrical and core specifications of a $100 \mathrm{~kW}$, $10 \mathrm{kHz}$ realized medium frequency transformer prototype. The employed transformer model is permeance-based and includes the model of hysteresis losses, evaluated inside the time domain simulations. Thereby, the electrical behavior of the transformer remains preserved with simulated core losses which are consistent with the analytical estimate. Furthermore, the simulated winding losses at rated power match well the measured ones.
\end{abstract}

\section{INTRODUCTION}

When designing a power converter system, the power engineers tend to utilize electric circuit simulations as a way of estimating the efficiency and the overall performance of the converter system in advance, i.e. already in the design phase. In this way, the invested design time can be shortened, which positively effects the overall cost. Nevertheless, such simulations need to include very accurate and physically intuitive models of the essential converter components, such as switching devices and magnetic components. Thereby, the goal is to combine all the important domains of a power converter system, such as electric, magnetic, dielectric, and thermal, in a common simulation environment.

Many analog electronic circuit simulation softwares exist to date and they are capable of accurately representing and modeling the exact working principles of power switching devices. Some of the examples are various versions of the SPICE software, such as ISPICE, LTspice, and PSPICE [1]-[3] and they mostly focus on a short time frame. On the contrary, system-level simulation softwares for electrical circuits, such as ANSYS Simplorer, Gecko, PLECS, PSIM, target significantly longer simulation times, mostly observing the general behavior of power converter components within a larger system. Furthermore, semiconductor manufacturers offer standardized data sheets containing important technical information of the device, such as rated values and switching characteristics. This data can be used by power electronic designers to understand the component's working principle and to be able to predict its behavior to a reliable degree, without having to perform additional tests on the device. Regarding standardized magnetic components, such as filter inductors and transformers, their data sheets mostly offer sparse information about the expected losses or the general design rules behind the component at hand. Additional data can be found in data sheets of magnetic core materials, which can be used for estimation of the corresponding losses. Nevertheless, manufacturers usually characterize the core material losses by using sinusoidal excitation, which in general does not apply to magnetic components employed at elevated frequencies within certain converter topologies (such as LLC resonant converter and Dual Active Bridge). When it comes to modeling magnetic components for simulation purposes, the models mostly rest on nonlinear hysteresis characterization achieved through mathematical models. Two main hysteresis models include the more complex Jiles-Atherton model [4], [5] and the Preisach model [6], whereas the Chan-Vladimirescu model [7] is used for core materials with symmetrical magnetization process, due to its simplicity.

This paper shows that based solely on the geometry of a certain magnetic component, and by knowing its electrical and material properties, it is possible to reliably calculate losses within a simulation environment. This is achieved by using recently developed magnetic models [8], [9], in order to accurately represent the magnetic hysteresis effect within the core. The magnetic model is based on the permeancecapacitance analogy and it is implemented with the help of the Preisach model. Moreover, in order to estimate the overall efficiency of a power converter system, prior to assembling the converter itself, semiconductor and winding losses are modeled and considered as well for various operating points. The modeling of winding losses is based on the application of the well-known Dowell's model. For verification purposes, a medium frequency transformer (MFT) prototype, developed 
in [10], with a known geometry and other design parameters, is modeled in a simulation environment as a part of an LLC converter with an ideal model and a magnetic hysteresis models. The core losses obtained in this way are further compared to analytically calculated losses, which were acquired with an improved version of the Steinmetz equation already during the design phase of the MFT prototype [11], [12].

Last but not least, the work presented in the paper offers a middle ground between a magnetic component's design and manufacturing by integrating a possibility to estimate different types of converter losses in a simulation environment. Furthermore, in this way a connection between the electrical and the magnetic domain in a single time domain simulation is achieved.

\section{Case Study: LlC Resonant Converter}

In order to demonstrate the integrated approach for loss estimation using simulations, a full-bridge LLC resonant converter is chosen as an exemplary topology for a power converter system. The corresponding electrical scheme is depicted in Figure 1. The converter comprises two full-bridge power stages, and an MFT with mounted resonant capacitors (marked with $C_{r 1}$ and $C_{r 2}$ ) in between, which acts as galvanic isolation between the power stages. As already mentioned, the transformer model is based on specifications of a $100 \mathrm{~kW}$, $10 \mathrm{kHz}$ MFT prototype, which is realized in [10]. The electrical specifications of the prototype are summarized in Table I. The first power stage is supplied with $750 \mathrm{~V}$ dc-link, whereas the second stage is used as a diode rectifier, since the power is transferred in a single direction. For LLC converters, it is common to use integrated transformers for galvanic isolation, i.e. to use the transformer leakage inductance as a series inductor, while the magnetizing inductance is used as a shunt inductor, which is usually, when modeled, placed across the primary winding, as visible from Figure 1. Together with the capacitors, they build a resonant network which filters higher harmonic currents. The resonant capacitor bank was realized as a series connection of multiple parallel ac film capacitors accounting to $37.5 \mu \mathrm{F}$. By selecting an adequate operating frequency, the already existing inductances are utilized to achieve zero voltage switching (ZVS), which is one of the main advantages of this converter type as it leads to low
Table I: Electrical specifications of the MFT prototype developed in [10].

\begin{tabular}{lccc}
\hline Electric Property & Label & Unit & Value \\
\hline Rated Power & $P_{\mathrm{n}}$ & $\mathrm{kW}$ & 100 \\
Primary Voltage & $V_{1}$ & $\mathrm{~V}$ & \pm 750 \\
Secondary Voltage & $V_{2}$ & $\mathrm{~V}$ & \pm 750 \\
Switching Frequency & $f_{\mathrm{sw}}$ & $\mathrm{kHz}$ & 10 \\
Leakage Inductance & $L_{\mathrm{r}}$ & $\mu \mathrm{H}$ & 8.4 \\
Magnetizing Inductance & $L_{\mathrm{m}}$ & $\mu \mathrm{H}$ & 750 \\
Resonant Capacitor & $C_{\mathrm{r}}$ & $\mu \mathrm{F}$ & 18.75 \\
Duty Cycle & $D$ & 1 & 0.5 \\
\hline
\end{tabular}

switching losses. The operating frequency is set depending on two characteristic resonant frequencies:

$$
\begin{aligned}
& f_{0}=\frac{1}{2 \pi \sqrt{L_{r} C_{r}}}=12.68 \mathrm{kHz}, \\
& f_{p}=\frac{1}{2 \pi \sqrt{\left(L_{r}+L_{m}\right) C_{r}}}=1.33 \mathrm{kHz} .
\end{aligned}
$$

Section IV provides more details about the influence of the operating frequency on the overall converter losses, i.e. semiconductor, core and winding losses. The transformer core was constructed from $48 \mathrm{U}$-shape (UU9316) magnetic core samples, made of ferrite material $C F 139$ and produced by Cosmo Ferrites Limited [13]. For simulation purposes, the IGBT module 5SNG 0150Q170300 is selected. Furthermore, the primary and secondary winding of the transformer each consist of eight turns (turns ratio of $1: 1$ ) of a square profiled copper Litz wire with 1400 strands (AWG 32). Precise information about the winding geometry is necessary for calculation of winding losses presented in Section III-B.

\section{A. Magnetic Modeling}

Depending on the exact applications, electrical properties of magnetic components like voltage (and current) vary in a wide range of values, from several volts (amperes) up to several hundreds of $\mathrm{kVs}(\mathrm{kAs})$. Nevertheless, the magnetic field quantities of a certain core material such as flux density $B$ and field strength $H$ remain limited due to core material properties, regardless of the size or power rating of the component. This fact allows to employ a core sample of a small

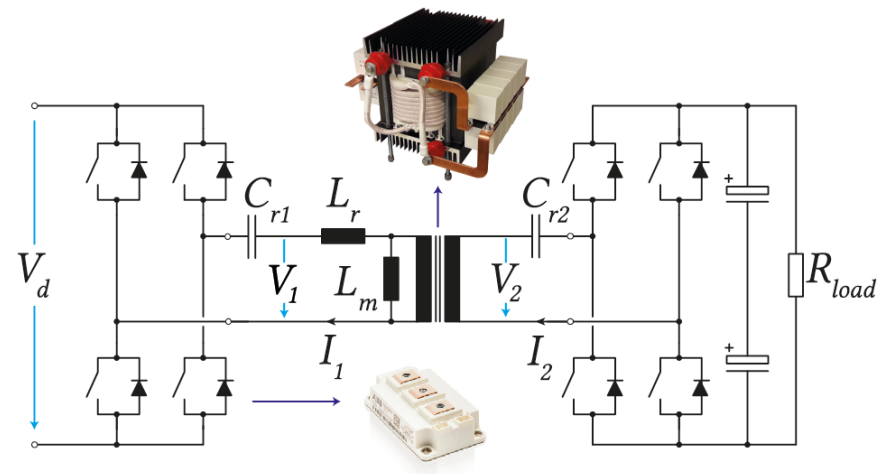

Figure 1: Electrical scheme of the LLC converter used for simulation with a graphical preview of the components employed in the experimental part.

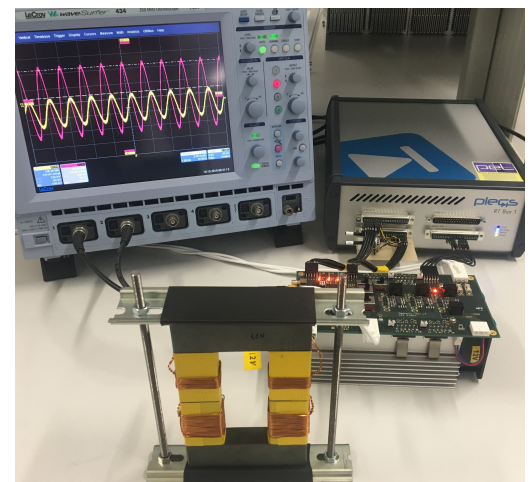

Figure 2: Magnetic characterization setup [9] used for the set of U-cores made of ferrite material CF139. 
size in order to reproduce the characteristic magnetization curve of the used material, even though the same material can be used for magnetic components designed for high voltage, high current applications. This lead to characterizing the core material of the MFT prototype with the help of a set of two U-shape cores, which are actually used in the MFT assembly. Furthermore, power losses of the core material are directly reflected by the material's hysteresis loop. Thereby, different operating conditions inevitably result in different shapes of magnetization curves on the $B-H$ plane.

Due to specificity of the ferrite material which is further addressed in Section III-A, the magnetic model, developed in [9], and used to simulate the MFT prototype is based on the frequency independent hysteresis effect. The model itself rests on the permeance-capacitance analogy, where any core shape can be represented by a magnetic circuit of permeances $\left(\mathscr{P}=\mu \frac{A}{l}\right)$. The parameters $A$ and $l$ reflect the geometry of a single core segment, i.e. the cross section area and the magnetic path length (MPL), and the permeability $\mu$ describes the non-linear magnetic nature of the core material, i.e. hysteresis. Thereby, the mathematical model of hysteresis from Preisach [6] is used and $\mu$ is modeled as a function of the magnetic field strength $(\mu(H))$. Finally, the purpose of the magnetic model is to correctly reproduce the hysteresis loop of the characterized material, i.e. estimate correctly the permeability.

The parametrization of the magnetic model in PLECS is performed through hysteresis measurements, which are taken with the help of characterization setup given in Figure 2 on a set of U-core made of the same ferrite material CF139, which is used for the MFT prototype. The primary winding is thereby excited by the power stage and the secondary winding is left open for voltage measurements. The turns number of the winding can be adapted to obtain the desired field strength and flux density range on the $B-H$ plane. Subsequently, the collected current and voltage measurements are converted to field strength $(H)$ and flux density $(B)$ inside the control unit through the following equations:

$$
H=\frac{N_{1} I}{l}, \quad B=\frac{\int V d t}{N_{2} A} .
$$

As already mentioned, the parameters $A$ and $l$ are geometry-

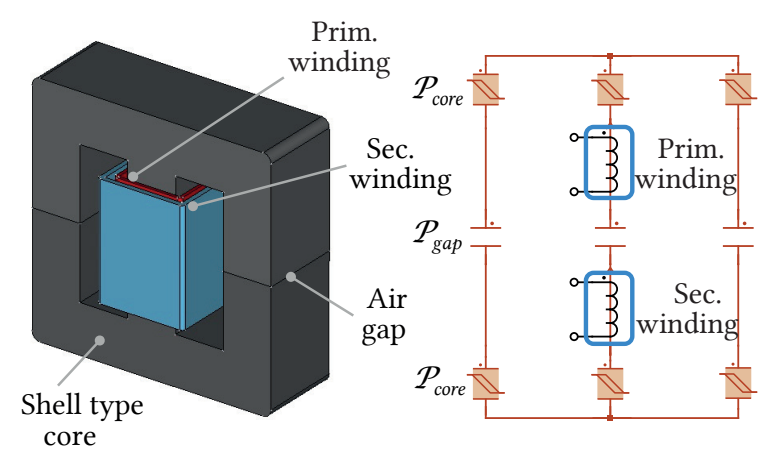

Figure 3: Transformer core represented by a set of permeances in a magnetic circuit. Figure is adopted from [14].

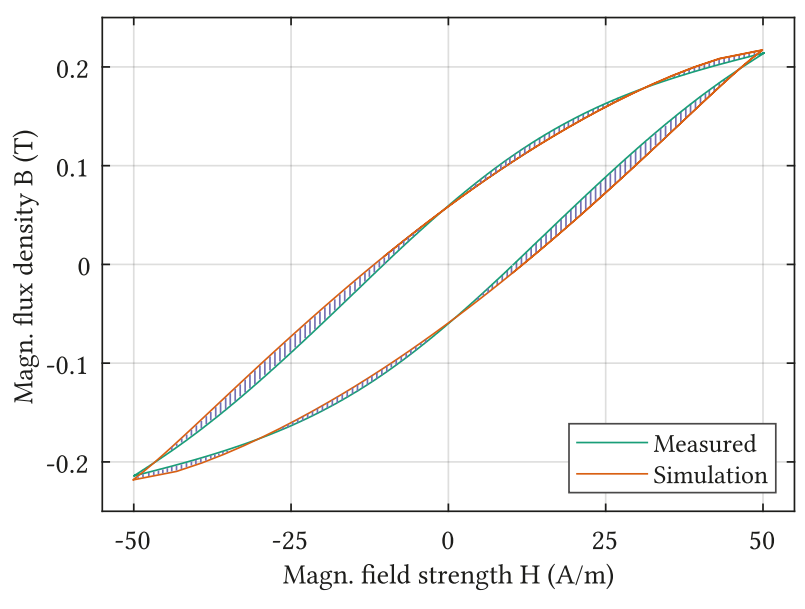

Figure 4: Comparison of the B-H curve measured at the test setup and the simulated curve resulting from the magnetic hysteresis model of the transformer core.

related parameters of a core segment, while $N_{1}$ and $N_{2}$ are the number of turns of the primary and the secondary winding, respectively.

As an end result of the performed core material characterization and the subsequent model parametrization a functional permeance block, based on the model of the frequency independent hysteresis effect, is obtained. Henceforth, the permeance block is used to model the existing MFT prototype in a simulation. Thereby, only the geometry (cross section area and MPL) of a respective core segment is changed and adjusted so to follow the overall MFT construction geometry, as depicted in Figure 3, where the magnetic circuit corresponding exactly to the MFT prototype and implemented in the simulation is depicted. Eventually, Figure 4 shows a good matching between the measured hysteresis loop of the core material and the one obtained as a result of modeling at no load conditions. The striped areas represent thereby the difference between the two hysteresis curves which leads to errors of per-cycle energy estimation. According to model verification conducted in [14] under different conditions and for variety of ferrite materials, the observed difference stays within the $10 \%$ overestimation range of the core losses when no energy is transferred.

\section{B. Semiconductor Losses}

To establish the base set of results, initial simulations with an ideal model of the MFT are conducted with the goal to replicate electrical properties of the existing test setup. The electrical circuit given in Figure 1 is simulated two times in PLECS depending on whether an ideal model of the transformer prototype is used, or the one based on magnetic hysteresis modeling, as presented in the section before. Considering the specific nature of the converter, no diode reverse recovery losses are expected. The same is valid for turn-on losses of the switching devices, due to the soft switching characteristic of a series resonant LLC converter. Figure 5 compares semiconductor losses obtained for both transformer models. The losses of the ideal model are shown with the filled bars, whereas the striped bars represent the losses of 


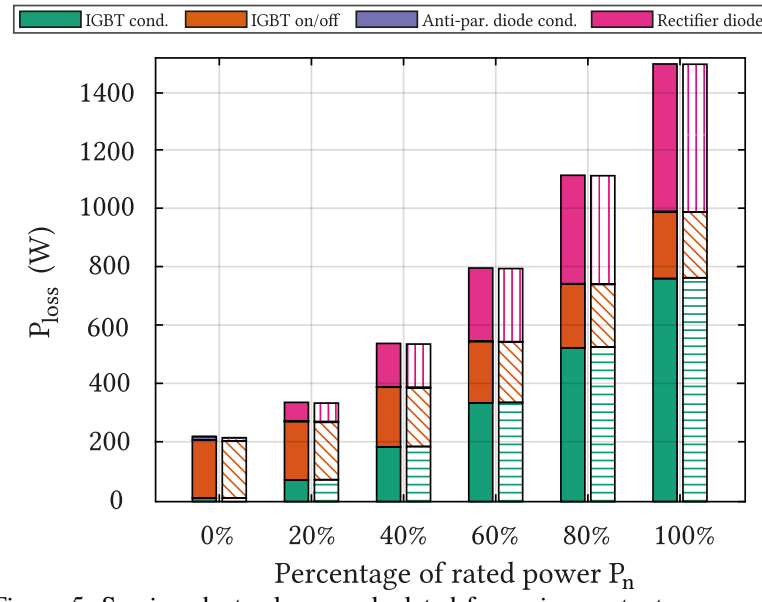

Figure 5: Semiconductor losses calculated for various output powers with an ideal transformer model (filled bars) and with a magnetic hysteresis model of the MFT (striped bars).

the detailed magnetic hysteresis model. The four presented types of semiconductor losses reflect the losses of all four devices in the inverter power stage, namely, conduction and switching devices of the four IGBTs and the conduction losses of the four corresponding anti-parallel diodes, as well as the losses of the four devices in the rectifier stage, where only diode conduction losses are observed (due to unidirectional power flow). The switching losses (including both on- and off-losses) of the IGBTs retain an approximately similar value regardless of the output power, whereas the conduction losses of both rectifier diodes and IGBTs increase with the increase of the transferred power, which is logical taking the primary and secondary current increase in Figure 6 into account. The figure shows both transformer currents and voltage waveforms simulated in the circuit from Figure 1 with an implemented magnetic hysteresis model of the MFT for various operating points. Considering that the switching frequency is selected below the resonant frequency $f_{0}$ calculated in (1), a half-cycle discontinuous conduction mode (HC-DCM) can be observed in the secondary current, which consequently allows the soft commutation of the rectifier diodes in the secondary stage. Lastly, the increased conduction losses of the anti-parallel diodes of the primary stage at no load conditions compared to the same type of losses for the increased power transfer can be explained by the prolonged time in which the anti-parallel diodes are conducting when no power is transferred. This can be observed in the first half-period for various primary currents given in the first plot of Figure 6. Clearly, the choice of model has no effect on the losses in the presented investigation. However, the ideal model of the transformer cannot be used to calculate magnetic core losses.

\section{Modeling of Medium Frequency Transformer Losses}

In general, the core and the winding losses are the two main contributors of total MFT losses. Therefore, their accurate estimation is important not only for performance and efficiency, but also for safe operation of the transformer.

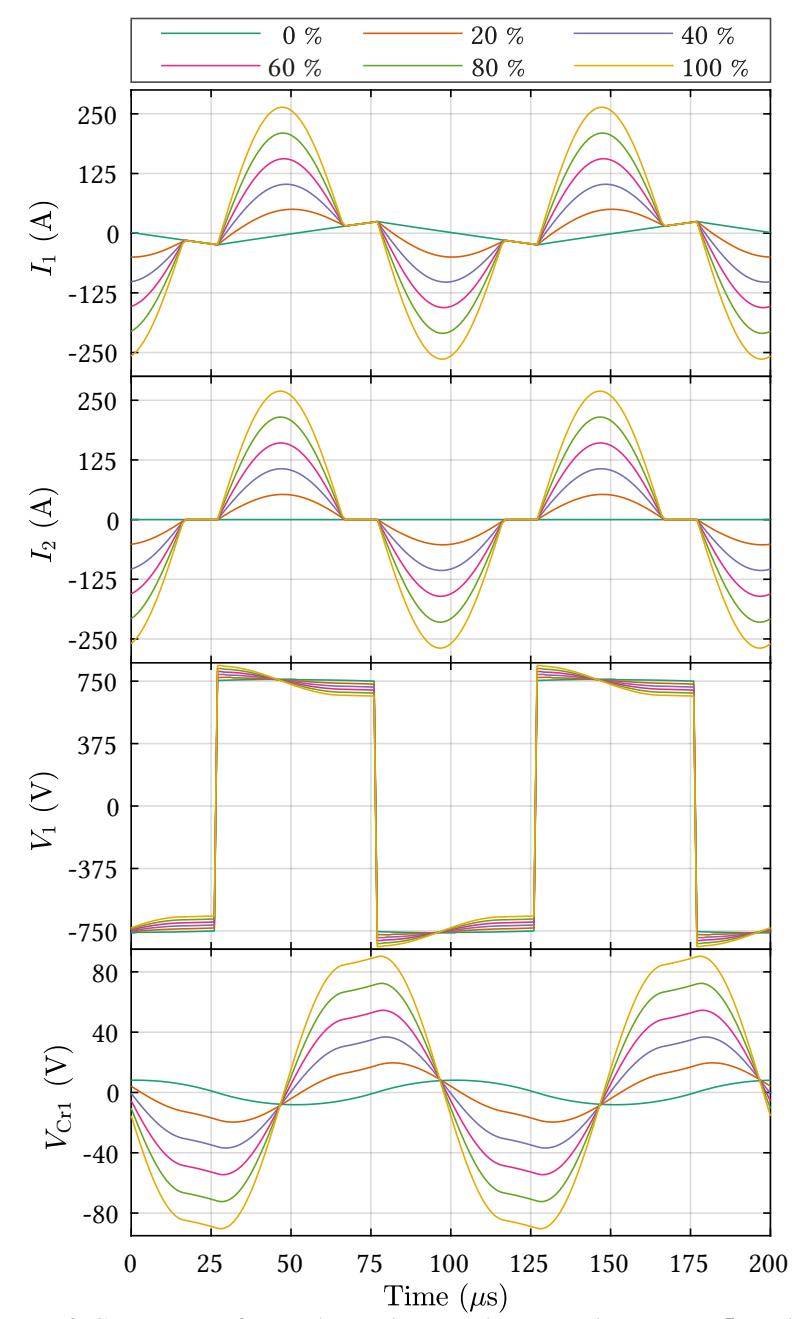

Figure 6: Current waveforms obtained in simulation on the primary $I_{1}$ and the secondary $I_{2}$ side, followed by primary voltage waveform $V_{1}$ and the voltage acquired at the capacitor bank $V_{\mathrm{Cr} 1}$ of the MFT at variable power ranging from $0 \%$ to $100 \%$.

\section{A. Core Losses}

Magnetic core losses can be principally divided into three parts as stated in [15]. Namely, frequency-independent hysteresis loss and frequency-dependent eddy current loss and relaxation loss. Nevertheless, due to the low electrical conductivity, eddy current losses can be neglected for ferrite materials [8]. According to [16] the relaxation losses are mostly visible for high-frequency (e.g. above $20 \mathrm{kHz}$ ) applications and for certain voltage waveforms (including zero phase voltages) applied to the magnetic component, which does not hold for an MFT within an LLC converter. Hence, it is assumed that the hysteresis effect generally dominates the core losses for the studied transformer prototype. Moreover, the core loss calculation can be performed: 1) analytically, based on the Steinmetz equation and its modified versions; 2) experimentally, with the help of calorimetric setups; 3 ) based on mathematical models of different magnetic effects within the material that cause core losses. In this paper, the power losses obtained with the help of magnetic hysteresis model are verified by the analytical approach. 
1) Analytical Approach: In order to extend the loss estimation to any non-sinusoidal excitation waveform, the improved generalized Steinmetz equation (IGSE) is used. The equation builds on the original Steinmetz equation

$$
P_{\mathrm{c}}=K f_{\mathrm{sw}}^{\alpha} B_{\mathrm{m}}^{\beta}
$$

with $K, \alpha$ and $\beta$ as the Steinmetz loss coefficients. They can be determined based on the core loss graphs depending on the magnetic flux density $B_{\mathrm{m}}$ and the excitation frequency $f_{\mathrm{sw}}$ provided in the core material data sheets. According to [12], the IGSE is determined by the following expression

$$
P_{\mathrm{c}}=\frac{1}{T} \int_{0}^{T} k_{\mathrm{i}}\left|\frac{d B(t)}{d t}\right|^{\alpha}(\Delta B)^{\beta-\alpha} d t,
$$

where $T$ represents the time period, $\Delta B$ stands for the peak-to-peak induction $\left(\Delta B=2 B_{\mathrm{m}}\right)$ and the coefficient $k_{i}$ is defined as

$$
k_{\mathrm{i}}=\frac{K}{2^{\beta+1} \pi^{\alpha-1}\left(0.2761+\frac{1.7061}{\alpha+1.354}\right)} .
$$

Considering a flux density waveform characteristic for an LLC resonant converter, a combination of (5) and (6) yields the following equation for the average power loss per unit of volume

$$
P_{\mathrm{c}}=2^{\beta} k_{\mathrm{i}} f_{\mathrm{sw}}^{\alpha} B_{\mathrm{m}}^{\beta}\left[D^{1-\alpha}+(1-D)^{1-\alpha}\right] .
$$

Parameters $D$ and $f_{\mathrm{sw}}$ are stated in Table I, whereas the flux density amplitude $B_{\mathrm{m}}$ is set to $0.21 \mathrm{~T}$, a value read off the hysteresis loop in Figure 4. Eventually, the core losses calculated analytically amount to around $225 \mathrm{~W}$.

2) Magnetic Hysteresis Approach: With a functional magnetic MFT model, the core losses are obtained by subtracting the power simulated at the secondary side from the primary side of the transformer. Eventually, Table II provides a visible agreement between the losses of the transformer core represented with the magnetic model and simulated at variable power levels and the analytically calculated value with the IGSE for the selected MFT design, core material and operating characteristics. Moreover, a certain decreasing trend can be recognized among the simulated values of the core losses, starting from a higher value obtained at no load conditions to the smallest core power loss among other operating points, simulated for the full rated power. This can be ascribed to the magnetic model, which responds to slightly different voltage excitation waveforms with slightly altered hysteresis loops. This eventually results in minor differences among the simulated power losses. The change in the shape of the voltage excitation depending on the operating point can be clearly seen in the primary transformer voltage shown in Figure 6.

Table II: Core losses acquired through simulations at the operating frequency of $10 \mathrm{kHz}$ for variable output powers.

\begin{tabular}{ccccccc}
\hline$P_{\mathrm{n}}$ & $0 \%$ & $20 \%$ & $40 \%$ & $60 \%$ & $80 \%$ & $100 \%$ \\
\hline$P_{\mathrm{c}}[W]$ & 229.6 & 226.4 & 223.8 & 221.2 & 218.6 & 216.1 \\
\hline
\end{tabular}

\section{B. Winding Losses}

At higher operating frequencies the Litz wire is commonly employed for current conduction, since it features intrinsically lower ac resistance compared to a single solid wire of the same cross-sectional area. This is due to drastic attenuation of the skin and the proximity effect, which get accentuated at higher operating frequencies thus increasing the resistance. However, the dc resistance of the Litz wire is higher for the same wire length, since each strand path is longer than the average wire length. It is generally assumed that eddy current densities caused by the two effects inside a specific layer are orthogonal, which makes the calculation of winding losses easier. In order to accurately estimate winding power losses a variation of the Dowell's model from [17] was used. The original model was developed for foil windings under two main assumptions:

- foil conductors occupy the entire core window height, assuring thereby one dimensional field $H_{\mathrm{y}}(x)$ along the core window,

- the use of magnetic core with infinite permeability.

Moreover, the model was based on the solution of electromagnetic equations in two dimensions. As a result, the model provides a frequency dependent expression for the ac resistance of the winding by introducing a resistance factor $F_{\mathrm{r}}$. In this way the winding loss contribution of higher current harmonics can be taken into account. Furthermore, to enable the application of the Dowell's model to square profiled Litz wires a porosity factor $\eta$ was introduced. Its purpose is to ensure that an equal magnetic field is generated along an enclosed path for the two different winding types and that the correct dc conductor resistance is provided. Correspondingly, the winding porosity factor represents the ratio of the actual layer copper area to the effective foil conductor area, and it is determined by the following

$$
\eta=\frac{m_{\mathrm{v}} d_{\mathrm{eq}}}{H_{\mathrm{w}}} \quad \text { with } \quad d_{\mathrm{eq}}=d \sqrt{\frac{\pi}{4}} .
$$

The parameter $m_{\mathrm{v}}$ stands for the equivalent number of vertical Litz layers in a winding, $d$ for the diameter of a single Litz strand, $d_{\mathrm{eq}}$ stands for the equivalent diameter of the squared strand, as well as the equivalent thickness of the foil winding.
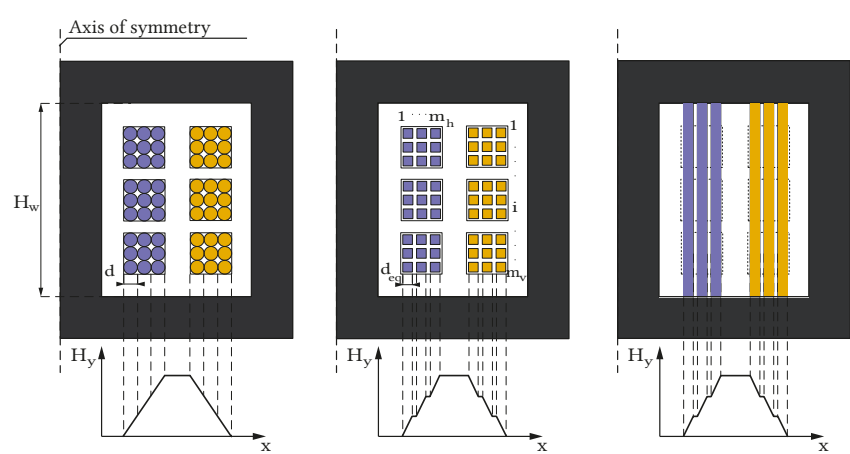

Figure 7: Winding equivalence with magnetic field distribution: (left) Squared Litz wire winding with round strands used in the MFT; (middle) equivalent squared Litz wire with square strands; (right) equivalent foil conductor covering the whole core window height. 
Lastly, the parameter $H_{\mathrm{w}}$ gives the height of the core window area, as can be seen from Figure 7. The figure shows the required adaptation of the winding structure, from the original MFT winding given on the left, to the equivalent foil conductors on the right, which represent the basis of the Dowell's winding model. Thereby, due to the existing vertical geometric symmetry of the shell transformer type, only the right half of the transformer is shown. Below each of the transformer halves a corresponding magnetic field in $x$ direction is given, assuring the same distribution is preserved. The total power loss experienced by a winding conducting the current $I$ is given by

$$
P_{\mathrm{win}}=\underbrace{\frac{\mathrm{MLT}}{\eta \sigma m_{\mathrm{h}} d_{\mathrm{eq}} H}}_{R_{\mathrm{DC}}} I_{\mathrm{DC}}^{2}+\sum_{n=1}^{\infty} R_{\mathrm{AC}, n} I_{\mathrm{RMS}, n}^{2} .
$$

The parameter $n$ stands for the harmonic order of the winding current, MLT is the mean length turn, $m_{h}$ is the equivalent number of horizontal Litz layers within the winding and $\sigma$ is the copper conductivity. According to Dowell, the ac resistance is defined by

$$
\begin{aligned}
R_{\mathrm{AC}}=F_{\mathrm{r}} R_{\mathrm{DC}} & =\Delta \frac{\sinh (2 \Delta)+\sin (2 \Delta)}{\cosh (2 \Delta)-\cos (2 \Delta)} R_{\mathrm{DC}}+ \\
& +\frac{2}{3}\left(m_{\mathrm{h}}^{2}-1\right) \Delta \frac{\sinh (\Delta)-\sin (\Delta)}{\cosh (\Delta)+\cos (\Delta)} R_{\mathrm{DC}}
\end{aligned}
$$

with the coefficient $\Delta=\frac{d_{\mathrm{eq}}}{\delta} \sqrt{\eta}$ known as the penetration ratio and the parameter $\delta$ describing the skin depth. A study conducted in [18] shows that high values of penetration ratio and high number of layers in a winding rapidly increase the resistance factor $F_{\mathrm{r}}$, i.e. the total winding resistance and consequently, the winding losses.

To ensure high accuracy, the harmonic content of both primary and secondary current was considered up to the $19^{\text {th }}$ order and the obtained winding losses are presented in Table III. Note that the Fourier transformation is performed during the simulation, so there is no need for post-processing. The value of the overall winding losses measured at the full rated power reported in [10] differs from the one obtained through simulation by less than $2 \%$. A non-zero value of the primary winding loss observed at no load conditions can be explained by the existing magnetizing current, shown in the first plot of Figure 6 (green curve labeled with $0 \%$ ). With the increased power transfer, the values for both primary and secondary winding losses expand in a similar fashion.

Table III: Primary and secondary winding losses acquired through simulations at variable transferred power.

\begin{tabular}{ccccccc}
\hline$P_{\mathrm{n}}$ & $0 \%$ & $20 \%$ & $40 \%$ & $60 \%$ & $80 \%$ & $100 \%$ \\
\hline$P_{\text {win,prim }}[\mathrm{W}]$ & 0.5 & 3 & 11.3 & 25.6 & 46.1 & 72.6 \\
\hline$P_{\text {win,sec }}[\mathrm{W}]$ & 0 & 2.9 & 11.8 & 26.7 & 47.7 & 74.9 \\
\hline$P_{\text {win,total }}[\mathrm{W}]$ & 0.5 & 5.9 & 23.1 & 52.3 & 93.7 & 147.5 \\
\hline
\end{tabular}

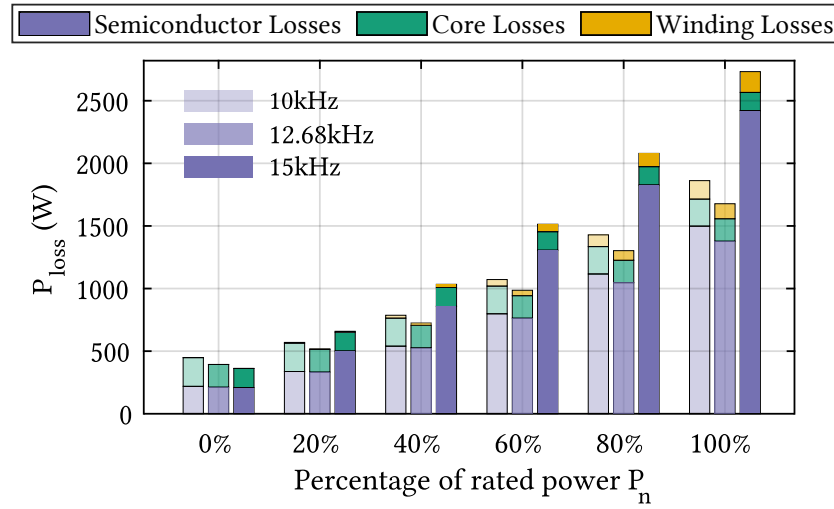

Figure 8: Semiconductor, core and winding losses simulated at three different operating frequencies $(10 \mathrm{kHz}, 12.68 \mathrm{kHz}$ and $15 \mathrm{kHz})$ at variable power ranging from $0 \%$ to $100 \%$ of the rated power. For each operating point the frequencies are arranged in the ascending order from left to right, which is also visible in the color shading of the bars.

\section{Overall Converter Losses}

Once all the major power loss sources within the converter have been identified and properly estimated, it is possible to discuss the overall converter losses and its efficiency for various operating frequencies. The frequencies are chosen in a way to represent the ability of the integrated approach to converter loss calculation, which includes the magnetic hysteresis model and the winding model, to function correctly in regions above and below the resonant frequency $f_{0}$, given in (1). Thus, the selected frequencies are $10 \mathrm{kHz}, 12.68 \mathrm{kHz}$ and $15 \mathrm{kHz}$. Core and winding losses are obtained in the same way as explained in Sections III-A and III-B. Note that regardless of the set frequency, the MFT prototype used for the transformer model is designed and optimized for $10 \mathrm{kHz}$.

Figure 8 shows in a summarized manner various converter losses (semiconductor, core and winding losses) simulated for the three selected operating frequencies at various loading conditions, ranging from $0 \%$ to $100 \%$ of the rated power. Regarding semiconductor losses a strong increase is observed for operation above resonance. This is especially due to trans-
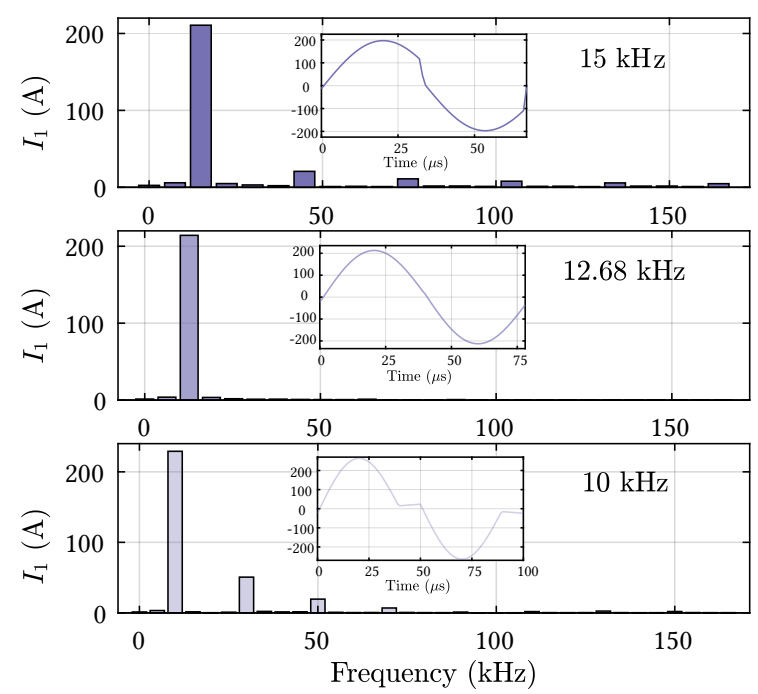

Figure 9: Harmonic content of the primary currents simulated at three different operating frequencies $(10 \mathrm{kHz}, 12.68 \mathrm{kHz}$ and $15 \mathrm{kHz})$ and rated power. 
ition from HC-DCM to continuous conduction mode (CCM), which implies higher values of primary turn-off currents than the value of the magnetizing current, which represents the turnoff current in HC-DCM. This can be well observed in Figure 9 which shows the time waveform and the corresponding harmonic content of the primary current at the rated power for the three operating frequencies. The increased turn-off current observed at $15 \mathrm{kHz}$ results in distinctly elevated IGBT switching losses which further grow with the increase of the transferred power. Nevertheless, the ZVS property of the converter stays preserved. According to Figure 8 the lowest semiconductor losses are observed at the resonant frequency. This is due to reduced amplitude of the transformer currents compared to the operation below resonance, as well as nonexistence of higher odd current harmonics visible for the operation both above and below resonance in Figure 9. Moreover, the transition point between the HC-DCM and CCM is exactly at the resonant frequency.

A distinct trend can be observed for the core losses regardless of the operating point and that is a loss decrease with the rising switching frequency. This is directly correlated with the size of the hysteresis loop, which tends to shrink in both directions as the operating frequency elevates. In spite of the fact that the core losses are determined by the product of the frequency and the enclosed area of the hysteresis, the reduction factor of the hysteresis loop area is bigger than the increase factor of the operating frequency, leading to smaller core losses at higher frequencies. Lastly, the winding losses show an expected rise in values for higher transferred powers regardless of the operating frequency. However, for each of the operating points the losses of both the primary and secondary winding are the lowest at the resonant frequency. Similarly to semiconductor losses, this can be well explained by the already mentioned reduction of currents amplitudes, and due to the fact that at the resonance point the transformer currents are closest to a pure sinusoidal current, which compared to other two frequencies contains less higher harmonics.

\section{Conclusion}

This paper proposes an integrated approach to loss calculation of a converter system, which includes semiconductor, magnetic core and winding losses. Analytically obtained core losses with the help of IGSE and the losses acquired through converter-level simulations based on a hysteresis model of a transformer coincide. The employed model preserves the electrical behavior of the MFT. In order to obtain the winding losses, the Dowell's model is chosen and successfully adjusted to the specific winding arrangement used in the MFT prototype. Eventually, the simulated winding losses at rated power differ by less than $2 \%$ of the measured losses, which confirms the validity of the selected model.

Finally, the ability to calculate semiconductor, core and winding losses in a common time-domain simulation environment offers the possibility to observe and estimate the overall efficiency of the converter system. Furthermore, such an approach provides an accurate insight in the designed converter system or the MFT before the actual construction takes place.

\section{Acknowledgment}

The results presented in this paper are a part of the EMPOWER project that has received funding from the European Research Council (ERC) under the European Union's Horizon 2020 research and innovation programme (Grant agreement No. 818706).

\section{REFERENCES}

[1] A. R. Hefner, "A dynamic electro-thermal model for the igbt," IEEE Transactions on Industry Applications, vol. 30, no. 2, pp. 394-405, 1994.

[2] C. L. Ma and P. O. Lauritzen, "A simple power diode model with forward and reverse recovery," in PESC'91 Record 22nd Annual IEEE Power Electronics Specialists Conference, IEEE, 1991, pp. 411-415.

[3] A. R. Hefner and D. L. Blackburn, "Simulating the dynamic electro-thermal behavior of power electronic circuits and systems," in [Proceedings] 1992 IEEE Workshop on Computers in Power Electronics, IEEE, 1992, pp. 143-151.

[4] D. C. Jiles and D. L. Atherton, "Theory of ferromagnetic hysteresis," Journal of magnetism and magnetic materials, vol. 61, no. 1-2, pp. 48-60, 1986.

[5] D. C. Jiles and J. Thoelke, "Theory of ferromagnetic hysteresis: Determination of model parameters from experimental hysteresis loops," IEEE Transactions on magnetics, vol. 25, no. 5, pp. 3928-3930, 1989.

[6] F. Preisach, "Über die magnetische Nachwirkung," Zeitschrift für Physik, vol. 94, no. 5-6, pp. 277-302, 1935.

[7] J. H. Chan, A. Vladimirescu, X.-C. Gao, P. Liebmann, and J. Valainis, "Nonlinear transformer model for circuit simulation," IEEE Transactions on Computer-Aided Design of Integrated Circuits and Systems, vol. 10, no. 4, pp. 476-482, 1991.

[8] M. Luo, D. Dujic, and J. Allmeling, "Modeling frequency-dependent core loss of ferrite materials using permeance-capacitance analogy for system-level circuit simulations," IEEE Transactions on Power Electronics, vol. 34, no. 4, pp. 3658-3676, 2018.

[9] M. Luo, D. Dujic, and J. Allmeling, "Modeling frequency independent hysteresis effects of ferrite core materials using permeance-capacitance analogy for system-level circuit simulations," IEEE Transactions on Power Electronics, vol. 33, no. 12, pp. 10055-10070, 2018.

[10] M. Mogorovic and D. Dujic, "100 kw, 10 khz mediumfrequency transformer design optimization and experimental verification," IEEE Transactions on Power Electronics, vol. 34, no. 2, pp. 1696-1708, 2018. 
[11] M. Mogorovic and D. Dujic, "Thermal modeling and experimental verification of an air cooled medium frequency transformer," in 2017 19th European Conference on Power Electronics and Applications (EPE'17 ECCE Europe), IEEE, 2017.

[12] K. Venkatachalam, C. R. Sullivan, T. Abdallah, and H. Tacca, "Accurate prediction of ferrite core loss with nonsinusoidal waveforms using only steinmetz parameters," in 2002 IEEE Workshop on Computers in Power Electronics, 2002. Proceedings., IEEE, 2002.

[13] Cosmo Ferrites Lim, https://www.cosmoferrites.com/.

[14] M. Luo, "Dynamic modeling of magnetic components for circuit simulation of power electronic systems," $\mathrm{PhD}$ thesis, EPFL, 2018.
[15] J. B. Goodenough, "Summary of losses in magnetic materials," IEEE Transactions on magnetics, vol. 38, no. 5, pp. 3398-3408, 2002.

[16] J. Muehlethaler, J. Biela, J. W. Kolar, and A. Ecklebe, "Improved core-loss calculation for magnetic components employed in power electronic systems," in IEEE Transactions on Power Electronics, vol. 27, 2012, pp. 964-973.

[17] P. Dowell, "Effects of eddy currents in transformer windings," Proceedings of the Institution of Electrical Engineers, vol. 113, no. 8, p. 1387, 1966.

[18] I. Villar, "Multiphysical characterization of mediumfrequency power electronic transformers," $\mathrm{PhD}$ thesis, EPFL, 2010. 